\title{
Staple arthroscopic fixation of tibial ACL avulsion with concomitant lobenhoffer approach for a posterolateral tibial plateau fracture
}

\begin{abstract}
Tibial eminence avulsion fracture or anterior cruciate ligament avulsion fractures are extremely uncommon, especially in adults. The objective of the treatment is to provide knee stability, allow early rehabilitation and reduce complications rate. Several methods for fracture fixation have been described in the literature. We report a case of a 39 year old male who presented with a severe tibial plateau fracture and an avulsed ACL which was arthroscopically fixated with a staple. This is a unique, simple and affordable technique with excellent clinical and functional results.
\end{abstract}

Keywords: ACL avulsion, staple fixation
Volume I2 Issue 5 - 2020

\author{
Filipa Gonçalves Pereira,' Francisco Brito,' \\ Tomás Osório,' Francisco Lemos,' Igor \\ Martins, ${ }^{2}$ André Ramos' \\ 'Orthopedics Service, Hospital José Joaquim Fernandes, Portugal \\ ${ }^{2}$ Orthopedics Service, Hospital Garcia de Orta, Portugal
}

\section{Correspondence: Filipa Gonçalves Pereira, Unidade Local de Saúde do Baixo Alentejo, Rua do Outeiral n 40, 47। 5 -142 Braga, Portugal, Tel 00351919010045,}

Email Filipa.pereira@ymail.com

\section{Abbreviations: ACL, anterior cruciate ligament}

\section{Introduction}

Tibial spine avulsion fracture is a rare injury, especially in skeletally mature patients. In literature this type of injury is mostly reported in pediatric population. ${ }^{1,2}$ The classical injury mechanism is due to hyperextension associated with a valgus or rotational force, usually secondary to a fall from a bicycle, a motor vehicle accident or associated with sports involving pivoting and axial load like ski or football. This leads to the avulsion of the tibial attachment of the ACL also known as tibial spine intercondylar fracture. ${ }^{3}$ High energy trauma is necessary to overcome the tensile bone strength at ACL attachment, therefore a higher incidence of associated injuries such as tibial plateau fractures and other ligaments tears is identified. ${ }^{2,4,5}$

The modified Meyers and McKeevers classification is the most commonly used (Figure 1). Type I nondisplaced fractures do not require surgical treatment, whereas Type III and IV are managed surgically. There is some debate about type II, but it is acknowledged that that a fragment displacement $<5 \mathrm{~mm}$ can be treated with close reduction and immobilization for 4 to 6 weeks. ${ }^{6}$

Several methods have been described for surgical treatment of tibial spine fractures. The most frequent techniques described in the literature are screw fixation with or without washer, suture fixation, wires and suture anchors but a gold standard treatment is yet to be determined..$^{7-9}$

The Goal of surgery is to achieve an adequate fracture reduction and fixation that restores ACL length, enables proper healing and early rehabilitation. ${ }^{10}$ The authors present a valid, affordable and easy accessed arthroscopic method of fixation for these type of fractures using a staple, with excellent functional and clinical results.

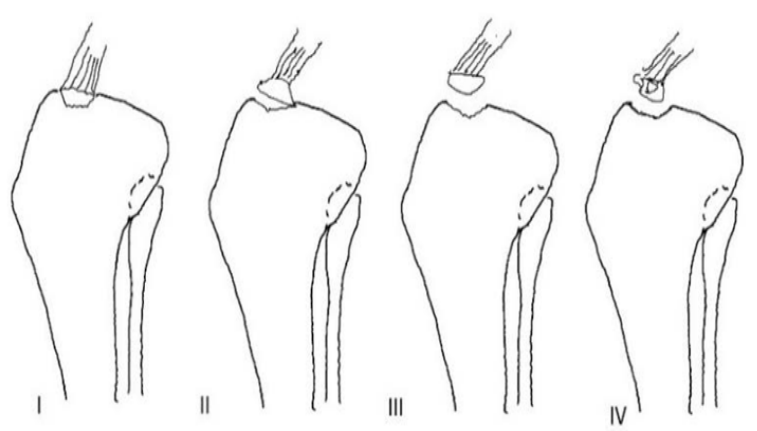

Figure I Modified Meyers and McKeever classification of tibial eminence fractures. Modified Meyers and McKeever classification. Figure (a) is a Type I and is non-displaced; (b) is a Type II which has a displaced anterior margin and hinged posterior cortex; (c) is a Type III and there is absence of any bony contact; (d) is a Type IV and is comminuted. Taken from Lubowitz JH, Elson WS, and Guttmann D, Part II: arthroscopic treatment of tibial plateau fractures: intercondylar eminence avulsion fractures Arthroscopy, 2005. 2 I (I): p. 86e92.

\section{Case presentation}

A 39 year old male presented after a motorcycle accident with swelling and impairment and disability of the left knee. Physical examination revealed a large knee effusion with intolerance to passive range of motion. Neurovascular injuries and compartment syndrome were excluded.

A preliminary orthogonal radiograph revealed a postero-lateral tibial plateau fracture and raised the suspicion of a displaced medial tibial spine anterior fracture (Figure 2). The patient underwent a CT-scan to further evaluate the fracture pattern (Figure 3) and was immediately immobilized with a long leg posterior cast with the knee in extension. 


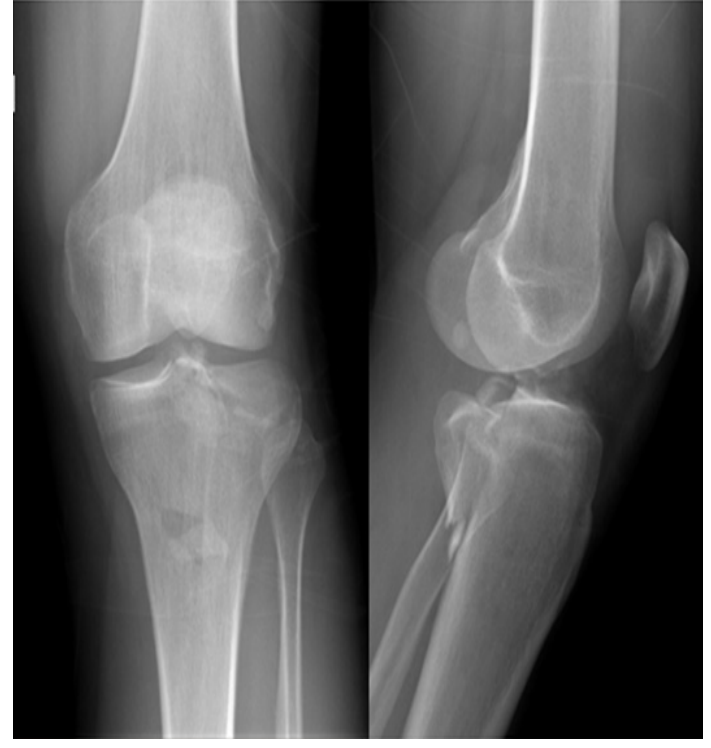

Figure 2 Left Knee $x$ ray two incidences.
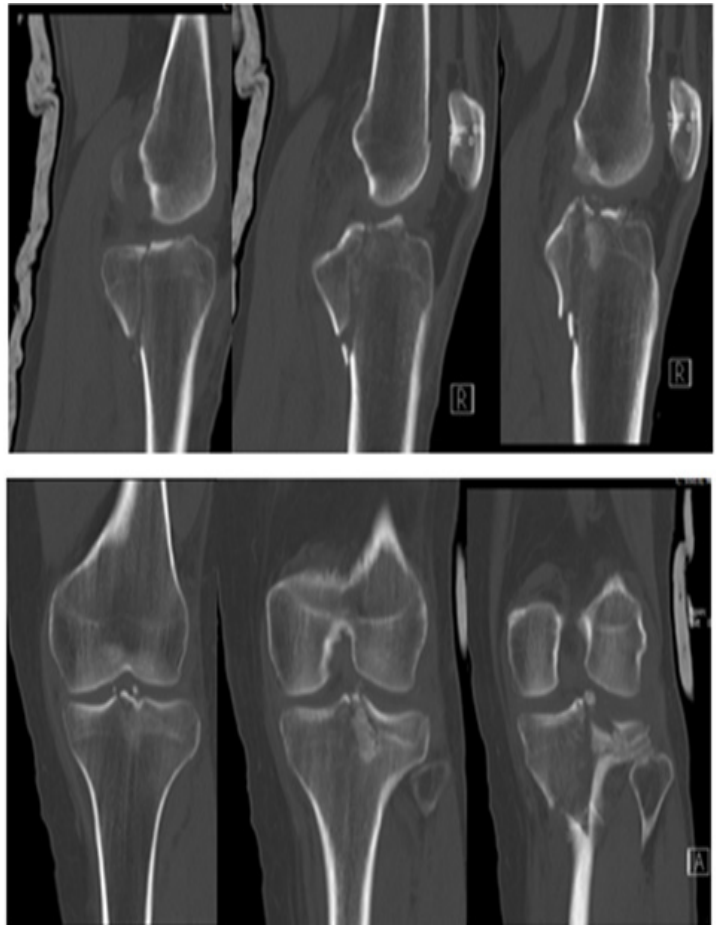

Figure 3 Left Knee CT-scan.

Six days after initial presentation, the patient underwent arthroscopic and open surgery

First step- A Lobenhoffer approach was performed to reduce and fixate the postero-lateral tibial plateau fragment with two $6.5 \mathrm{~mm}$ cannulated screws with washer and an anti-gliding $3.5 \mathrm{~mm}$ plate. This approach was selected due to the fracture pattern and also to the fact that reduction is achieved with hiper-extension.

Second step- Arthroscopy was performed for tibial plateau reduction, evaluation and fixation of tibial spine avulsion fracture. A large osseous fragment was found attached to ACL (Figure 4), with no comminution. No meniscal or intermeniscal ligament tissue was found in the fracture bed. There were no meniscal nor PCL injuries.
Maintaining the knee in $90^{\circ}$ of flexion, the medial tibial spine was temporarily stabilized with $\mathrm{K}$ wires percutaneously and fixated with a $10 \mathrm{~mm}$ staple trough the antero-medial portal that was created in more superior position to provide a greater angle for the introduction of the staple handle (Figure 5).
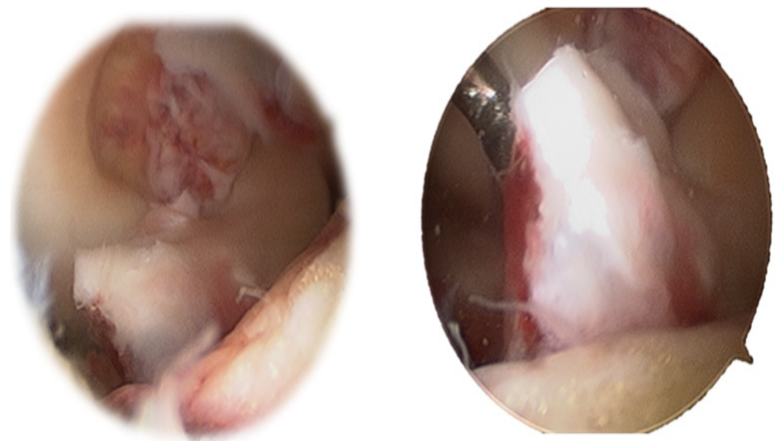

Figure 4 Arthroscopic view of avulsed LCA insertion with a large bony fragment.

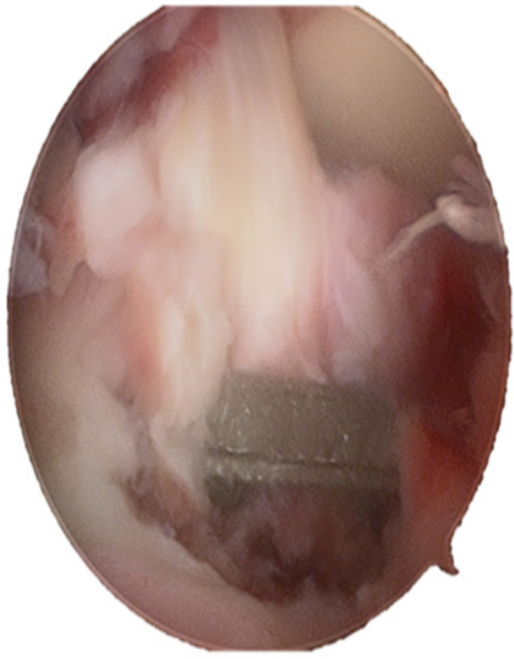

Figure 5 Staple $\mathrm{ACL}$ arthroscopic fixation.

\section{Postoperative radiograph confirmed satisfactory anatomic fracture reduction (Figure 6)}

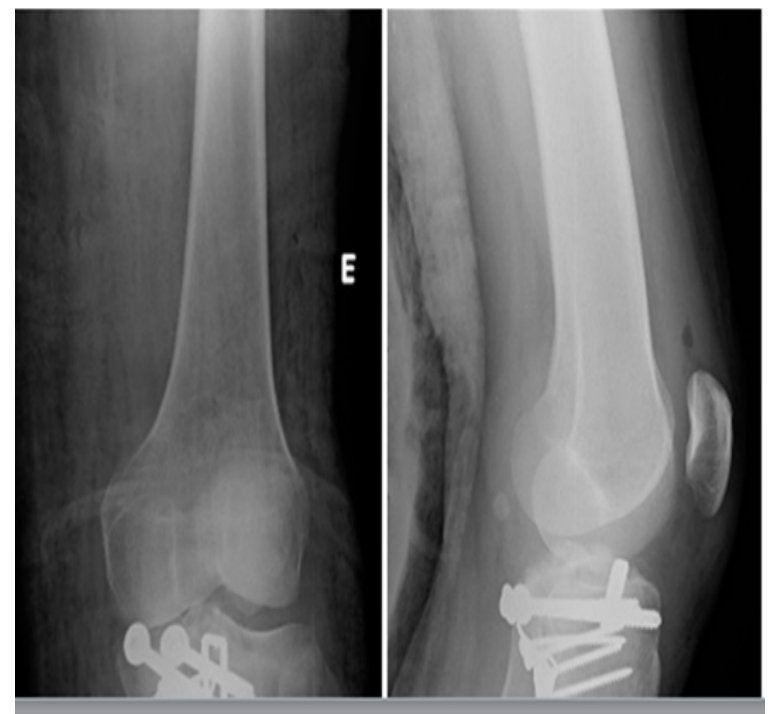

Figure 6 Postoperative orthogonal $x$-ray. 
The Patient was immobilized with long leg cast for 4 weeks. At the time of removal, he was showing signs of knee stiffness $\left(-5^{\circ}\right.$ of extension and $60^{\circ}$ of flexion) and thigh muscle atrophy. An intensive rehabilitation program was started with isometric and closed chain exercises, with partial weight bearing in the first 2 weeks after cast removal.

Radiological union was achieved 8 weeks following surgery (Figure 7).
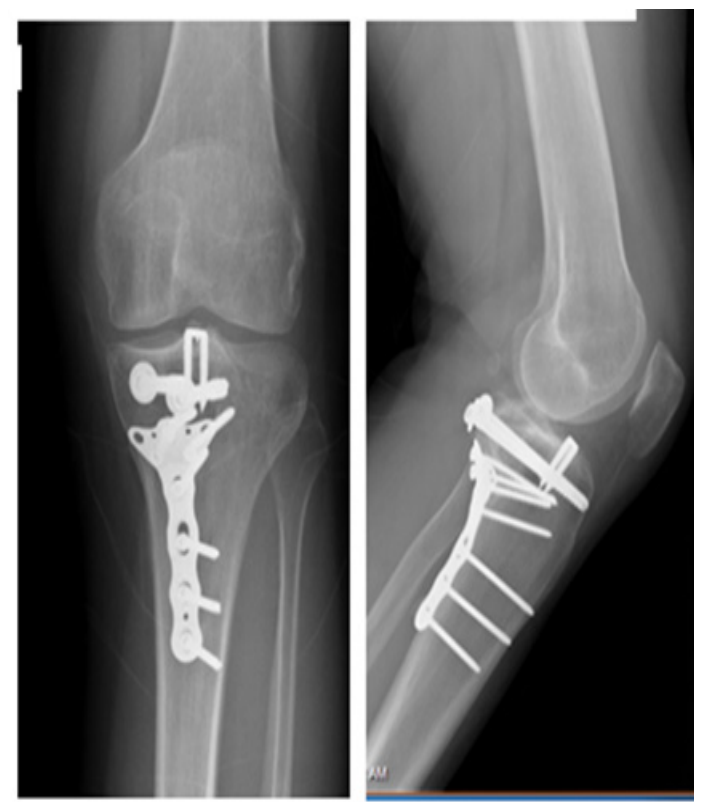

Figure 78 weeks postoperative orthogonal knee x-ray.

One year after surgery the patient had no limitations in his daily activities, no pain or subjective instability and had returned to his previous activity level. He recovered full range of motion and had a Lysholm score of 91 (Figure 8 \&9).

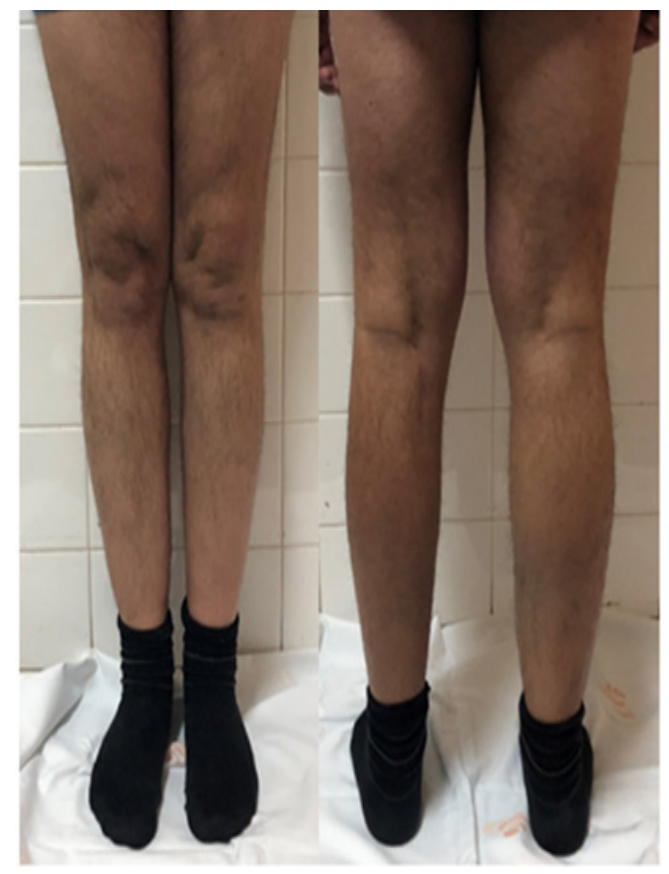

Figure 8 Full extension of both knees.

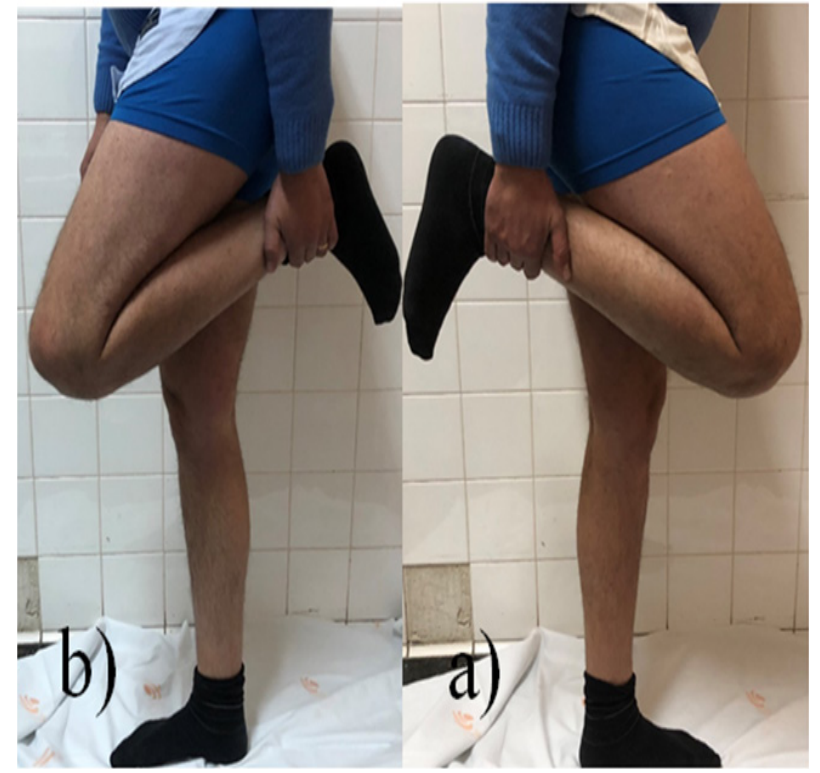

Figure 9 a) left knee flection; b) right knee flection.

\section{Discussion}

Tibial spine fractures typically occur at the distal insertion of the ACL (antero-laterally to the medial tibial spine). Radiographs and CT scan can be useful for the diagnosis but due to its location, frequent surrounding tissues in the fracture bed, and high frequency of associated injuries in adults, MRI can be a major advantage in this type of injuries. ${ }^{11}$

The authors consider the use of MRI of extreme value. Unfortunately, in this case de MRI could not be performed at in useful time.Despite lack of consensus regarding the treatment of tibial spine avulsion fractures, an arthroscopic approach has some known advantages compared to an arthrotomy because it is less invasive and has a lower stiffness risk. Additionally, arthroscopy allows a more accurate approach of the fracture, direct reduction control, evaluation and treatment of associated injuries, early mobilization, reduced hospitalization time and reduced morbidity. ${ }^{12}$

Direct visualization is of utmost importance, since the reduction can be blocked by the interposition of the inter-meniscal ligament or the anterior horn of medial meniscus.In addition, fixation methods can be controversial. Mechanical studies have shown that suture fixation and suture anchor have a higher load to failure and survival rate during cyclic loading compared to screw fixation. ${ }^{13}$

In this case the authors describe the use of a staple, placed arthroscopically to fixate the avulsed LCA. The medial tibial spine fracture line identified extended anteriorly up to tibial border. Therefore, the staple placed in the anterior part of fragment could not interfere with femur extension. This technique can be performed without specific arthroscopic instruments and is also an affordable option compared to the others with similar clinical and functional scores. ${ }^{14}$

Disadvantages of the use of staple fixation include the risk of iatrogenic fracture while hammering, articular surface damage during its placement, increased pull out failure compared with suture fixation and increased rate of hardware removal.In the presence of small or several fragments a secure fixation may be compromised with the 
use of a single staple, so careful assessment of preoperative images is required.

The most common complication is ACL laxity either if the patient is treated surgically or nonsurgically, with positive Lachman test in 20$60 \%$ of patients and positive Pivot Shift test in $8-40 \%$. An association with the laxity postoperatively and the severity of the injury has been documented. ${ }^{8,15}$

Even though subjective and functional instability are not often observed, since residual laxity is well tolerated, nearly $20 \%$ of patients will need an ACL reconstruction. ${ }^{16,17}$ Another common complication is arthrofibrosis with an incidence rate of $10-29 \%$, primarily related to immobilization, concomitant ACL rupture and surgery time exceeding 2hours. ${ }^{12,18,19}$ No correlation was found between the type of fixation and knee stiffness in literature. ${ }^{1,8}$ Risk Factors for postoperative stiffness include long immobilization period, complete fracture displacement, malunion, hardware protrusion, more than one surgical intervention, ${ }^{20}$ and increasing age. ${ }^{21,22}$

In the presented case, the patient was immobilized for 4 weeks, as recommended in several rehabilitation protocols. In the authors' opinion, immobilization time could have been reduced, since the fracture fixation was strong and earlier mobilization might have prevented the initial stiffness. In this particular case weight bearing could not be started earlier because of the tibial plateau fracture. ${ }^{6,10}$

\section{Conclusion}

Tibial spine fractures are uncommon injuries and usually associated with secondary injuries, especially in skeletally mature patients.The approach of these fractures is still controversial, as well as the fixation method used. Gold standard treatment is yet to be determined but the goal is to have an adequate reduction, ACL tension and secure fixation in order to start early and aggressive postoperative rehabilitation and reduce complications. In the authors' opinion, arthroscopic staple fixation is a simple and effective option with favorable clinical and functional results.

\section{Acknowledgments}

Dr. André Lopes Vasques, Catarina Amorim

\section{Conflicts of interest}

The authors declare no conflict of interest regarding the publication of this article.

\section{Funding}

None.

\section{References}

1. Edmonds EW, Fornari ED, Dashe J, et al. Results of displaced pediatric tibial spine fractures: A comparison between open arthroscopic, and closed management. JP ediatr Orthop. 2015;35(7):651-656.

2. Anderson CN, Anderson AF. Tibial eminence fracture. Clin Sports Med. 2011;30(4):727-742.

3. Lubowitz JH, Elson WS, Guttmann D. Part II: Arthroscopic treatment of tibial plateau fractures. Intercondylar eminence avulsion fractures. Arthroscopy. 2005;21(1):86-92.
4. Sapre V, Dwidmuthe SC, Bagaria V, et al. Functional outcome in tibial spine fracture treated with arthroscopic pull through suture technique. $J$ Orthop Traumatol Rehabil. 2015;8:6-10 .

5. Stevens MA, El-Khoury G, Kathol MH, et al. Imaging features of avulsion injuries. Radio Graphics. 1999;19(3):655-672 .

6. Strauss EJ, Kaplan DJ, Weinberg ME, et al. Arthroscopic Management of Tibial Spine Avulsion Fractures: Principles and Techniques. $J$ Am Acad Orthop Surg. 2018;26(10):360-367.

7. Bogunovic L, Tarabichi M, Harris D, et al. Treatment of tibial eminence fractures: a systematic review. J Knee Surg. 2015;28(3):255-262.

8. Gans I, Baldwin KD, Ganley TJ. Treatment and Management Outcomes of Tibial Eminence Fractures in Pediatric Patients: A Systematic Review. Am J Sports Med. 2014;42(7):1743-1750.

9. Shin YW, Uppstrom TJ, Haskel JD, et al. The tibial eminence fracture in skeletally immature patients. Curr Opin Pediatr. 2015;27(1):50-57.

10. Indranil Kushare, Rushyuan Jay Lee, Henry B Ellis Jr, et al. Tibial Spine Fracture Management - Technical Tips and Tricks from the Tibial Spine Fracture Research Interest Group. JPOSN. 2020;2(1):1-9.

11. Ishibashi Y, Rudy TW, Livesay GA, et al. The effect of anterior cruciate ligament graft fixation site at the tibia on knee stability: Evaluation using a robotic testing system. Arthroscopy. 1997;13(2):177-182.

12. Coyle C, Jagernauth S, Ramachandran M: Tibial eminence fractures in the paediatric population: A systematic review. J Child Orthop. 2014;8(2):149-159.

13. Anderson CN, Nyman JS, McCullough KA, et al. Biomechanical evaluation of physeal sparing fixation methods in tibial eminence fractures. Am J Sports Med. 2013;41(7):1586-1594.

14. Sundararajan SR, Rajasekaran S, Bernard SL. Displaced anterior cruciate ligament avulsion fractures: Arthroscopic staple fixation. Indian J Orthop. 2011;45(4):324-329.

15. Sapre V, Bagaria V. Tibial spine avulsion fractures: Current concepts and technical note on arthroscopic techniques used in management of these injuries. Regional Arthroscopy. 2013.

16. Kocher MS, Foreman ES, Micheli LJ. Laxity and functional outcome after arthroscopic reduction and internal fixation of displaced tibial spine fractures in children. Arthroscopy. 2003;19(10):1085-1090.

17. Willis RB, Blokker C, Stoll TM, et al. Long-term follow-up of anterior tibial eminence fractures. J Pediatr Orthop. 1993;13(3):361-364.

18. Vander Have KL, Ganley TJ, Kocher MS, et al. Arthrofibrosis after surgical fixation of tibial eminence fractures in children and adolescents. Am J Sports Med. 2010;38(2):298-301.

19. Parikh SN, Myer D, Eismann EA. Prevention of arthrofibrosis after arthroscopic screw fixation of tibial spine fracture in children and adolescents. Orthopedics. 2014;37(1):e58-e65.

20. Watts CD, Larson AN, Milbrandt TA. Open versus arthroscopic reduction for tibial eminence fracture fixation in children. $J$ Pediatr Orthop. 2016;36(5):437-439.

21. Aderinto J, Walmsley P, Keating JF. Fractures of the tibial spine: Epidemiology and outcome. The Knee. 2008;15(3):164-167.

22. Song EK, Seon JK, Park SJ, et al. Clinical outcome of avulsion fracture of the anterior cruciate ligament between children and adults. $J$ Pediatr Orthop B. 2009;18(6):335-338. 